\title{
Low serum albumin associated with worse transplant outcome
}

A new study published in the American Journal of Transplantation reports that renal transplant recipients with lower serum albumin levels before transplantation have worse outcomes after transplantation than those with higher levels.

Many studies have investigated predictors of mortality and graft failure following renal transplantation, but few studies have looked at the association between various parameter values obtained during pretransplantation dialysis therapy and post-transplantation outcomes. Low serum albumin levels in patients on dialysis are indicative of more-severe comorbidity and worse general health status and are associated with cardiovascular and all-cause mortality across the entire range of chronic kidney disease. However, it is unknown whether low serum albumin levels during dialysis influence posttransplantation outcomes. Molnar and colleagues hypothesized that lower pretransplantation albumin levels would be associated with worse post-transplantation outcomes.

The researchers used data from 8,961 kidney transplant recipients who were included in the Scientific Registry of Transplant Recipients and had received maintenance hemodialysis at an outpatient dialysis facility in the USA from July 2001 to June 2006.

\section{The association between pretransplant low serum albumin and higher mortality was strictly linear and incremental 77}

The patients were categorized into five groups on the basis of their average serum albumin level over the 3 months before transplantation $(<37.7 \mathrm{~g} / \mathrm{l}, 37.7-39.6 \mathrm{~g} / \mathrm{l}$, 39.7-41.2 g/l, 41.3-42.9 g/l and $\geq 43.0 \mathrm{~g} / \mathrm{l}$ ). Cox regression analyses adjusted for multiple factors including case-mix and transplant-related variables, showed that each $2 \mathrm{~g} / \mathrm{l}$ increase in serum albumin level before transplantation was associated with a $13 \%$ reduction in the risk of all-cause mortality, a $17 \%$ decrease in the risk of cardiovascular mortality, and a $7 \%$ decrease in the combined risk of death or graft failure over the 6-year observation period.

Further analyses showed that patients whose pretransplantation serum albumin level was in the highest quintile had a 53\% lower risk of all-cause death, a $74 \%$ lower risk of cardiovascular death, and a 32\% reduction in the combined risk of all-cause death or graft failure than patients with serum albumin levels in the lowest quintile.

Multivariate logistic regression analyses showed that each $2 \mathrm{~g} / \mathrm{l}$ increase in pretransplantation serum albumin level was associated with a $4 \%$ decrease in the risk of post-transplantation delayed graft function, defined as the need for at least one dialysis session in the first week after kidney transplantation.

"The association between pretransplant low serum albumin and higher mortality was strictly linear and incremental," conclude the authors. "If our findings are verified in additional studies, assessment of nutritional status and comorbid conditions in waitlisted patients may become an important task in [providing] care to transplant waitlisted patients."

Rebecca Ireland

Original article Molnar, M. Z. et al. Associations of pretransplant serum albumin with post-transplant outcomes in kidney transplant recipients. Am. J. Transplant. doi:10.1111/j.1600-6143.2011.03480.x 